\title{
Statistical convergence through de la Vallée-Poussin mean in locally solid Riesz spaces
}

\author{
Syed Abdul Mohiuddine ${ }^{1 *}$, Abdullah Alotaibi ${ }^{1}$ and Mohammad Mursaleen²
}

\section{"Correspondence:}

mohiuddine@gmail.com

'Department of Mathematics,

Faculty of Science, King Abdulaziz

University, P.O. Box 80203, Jeddah,

21589, Saudi Arabia

Full list of author information is

available at the end of the article

\begin{abstract}
The notion of statistical convergence was defined by Fast (Colloq. Math. 2:241-244, 1951) and over the years was further studied by many authors in different setups. In this paper, we define and study statistical $\tau$-convergence, statistically $\tau$-Cauchy and $S^{*}(\tau)$-convergence through de la Vallée-Poussin mean in a locally solid Riesz space. MSC: 40A35; 40G15; 46A40

Keywords: statistical convergence; statistical Cauchy; de la Vallée-Poussin mean; locally solid Riesz space
\end{abstract}

\section{Introduction and preliminaries}

Since 1951, when Steinhaus [1] and Fast [2] defined statistical convergence for sequences of real numbers, several generalizations and applications of this notion have been investigated. For more detail and related concepts, we refer to [3-29] and references therein. Quite recently, Di Maio and Kǒcinac [30] studied this notion in topological and uniform spaces and Albayrak and Pehlivan [31], and Mohiuddine and Alghamdi [32] for real and lacunary sequences, respectively, in locally solid Riesz spaces. Afterward, the idea was extended to double sequences by Mohiuddine et al. [33] in the framework of locally solid Riesz spaces.

Let $K$ be a subset of $\mathbb{N}$, the set of natural numbers. Then the asymptotic density of $K$ denoted by $\delta(K)$ is defined as

$$
\delta(K)=\lim _{n \rightarrow \infty} \frac{1}{n}|\{k \leq n: k \in K\}|,
$$

where the vertical bars denote the cardinality of the enclosed set.

The number sequence $x=\left(x_{j}\right)$ is said to be statistically convergent to the number $\ell$ if for each $\epsilon>0$,

$$
\lim _{n \rightarrow \infty} \frac{1}{n}\left|\left\{j \leq n:\left|x_{j}-\ell\right| \geq \epsilon\right\}\right|=0 .
$$

In this case, we write $s t-\lim x_{j}=\ell$.

\section{Springer}

(O) 2013 Mohiuddine et al.; licensee Springer. This is an Open Access article distributed under the terms of the Creative Commons Attribution License (http://creativecommons.org/licenses/by/2.0), which permits unrestricted use, distribution, and reproduction in any medium, provided the original work is properly cited. 
Remark 1.1 It is well known that every statistically convergent sequence is convergent, but the converse is not true. For example, suppose that the sequence $x=\left(x_{n}\right)$ is defined as

$$
x=\left(x_{n}\right)= \begin{cases}\sqrt{n} & \text { if } n \text { is a square } \\ 0 & \text { otherwise. }\end{cases}
$$

It is clear that the sequence $x=\left(x_{n}\right)$ is statistically convergent to 0 , but it is not convergent.

Now we recall some definitions related to the notion of a locally solid Riesz space. Let $X$ be a real vector space and $\leq$ be a partial order on this space. Then $X$ is said to be an ordered vector space if it satisfies the following properties:

(i) If $x, y \in X$ and $y \leq x$, then $y+z \leq x+z$ for each $z \in X$.

(ii) If $x, y \in X$ and $y \leq x$, then $\lambda y \leq \lambda x$ for each $\lambda \geq 0$.

If in addition $X$ is a lattice with respect to the partial order $\leq$, then $X$ is said to be a Riesz space (or a vector lattice) [34].

For an element $x$ of a Riesz space $X$, the positive part of $x$ is defined by $x^{+}=x \vee \theta=$ $\sup \{x, \theta\}$, the negative part of $x$ by $x^{-}=(-x) \vee \theta$ and the absolute value of $x$ by $|x|=x \vee(-x)$, where $\theta$ is the zero element of $X$.

A subset $S$ of a Riesz space $X$ is said to be solid if $y \in S$ and $|x| \leq|y|$ imply $x \in S$.

A topological vector space $(X, \tau)$ is a vector space $X$ which has a (linear) topology $\tau$ such that the algebraic operations of addition and scalar multiplication in $X$ are continuous. The continuity of addition means that the function $f: X \times X \rightarrow X$ defined by $f(x, y)=x+y$ is continuous on $X \times X$, and the continuity of scalar multiplication means that the function $f: \mathbb{R} \times X \rightarrow X$ defined by $f(\lambda, x)=\lambda x$ is continuous on $\mathbb{R} \times X$.

Every linear topology $\tau$ on a vector space $X$ has a base $\mathcal{N}$ for the neighborhoods of $\theta$ satisfying the following properties:

$\left(C_{1}\right)$ Each $Y \in \mathcal{N}$ is a balanced set, that is, $\lambda x \in Y$ holds for all $x \in Y$ and every $\lambda \in \mathbb{R}$ with $|\lambda| \leq 1$.

$\left(C_{2}\right)$ Each $Y \in \mathcal{N}$ is an absorbing set, that is, for every $x \in X$, there exists $\lambda>0$ such that $\lambda x \in Y$.

$\left(C_{3}\right)$ For each $Y \in \mathcal{N}$, there exists some $E \in \mathcal{N}$ with $E+E \subseteq Y$.

A linear topology $\tau$ on a Riesz space $X$ is said to be locally solid (cf. [35, 36]) if $\tau$ has a base at zero consisting of solid sets. A locally solid Riesz space $(X, \tau)$ is a Riesz space equipped with a locally solid topology $\tau$.

In this paper, we define and study statistical $\tau$-convergence, statistically $\tau$-Cauchy and $S^{*}(\tau)$-convergence through de la Vallée-Poussin mean in a locally solid Riesz space.

\section{Generalized statistical $\tau$-convergence}

Throughout the text, we write $\mathcal{N}_{\text {sol }}$ for any base at zero consisting of solid sets and satisfying the conditions $\left(C_{1}\right),\left(C_{2}\right)$ and $\left(C_{3}\right)$ in a locally solid topology. The following idea of $\lambda$-statistical convergence was introduced in [37] and further studied in [38-40].

Let $\lambda=\left(\lambda_{n}\right)$ be a non-decreasing sequence of positive numbers tending to $\infty$ such that

$$
\lambda_{n+1} \leq \lambda_{n}+1, \quad \lambda_{1}=0 .
$$


The generalized de la Vallée-Poussin mean is defined by

$$
t_{n}(x)=: \frac{1}{\lambda_{n}} \sum_{j \in I_{n}} x_{j}
$$

where $I_{n}=\left[n-\lambda_{n}+1, n\right]$.

A sequence $x=\left(x_{j}\right)$ is said to be $(V, \lambda)$-summable to a number $\ell$ if

$$
t_{n}(x) \rightarrow \ell \quad \text { as } n \rightarrow \infty
$$

A sequence $x=\left(x_{j}\right)$ is said to be strongly $(V, \lambda)$-summable to a number $\ell$ if

$$
\frac{1}{\lambda_{n}} \sum_{j \in I_{n}}\left|x_{j}-\ell\right| \rightarrow 0 \quad \text { as } n \rightarrow \infty
$$

We denote it by $x_{j} \rightarrow \ell[V, \lambda]$ as $j \rightarrow \infty$.

Let $K \subseteq \mathbb{N}$ be a set of positive integers, then

$$
\delta_{\lambda}(K)=\lim _{n \rightarrow \infty} \frac{1}{\lambda_{n}}\left|\left\{n-\lambda_{n}+1 \leq j \leq n: j \in K\right\}\right|
$$

is said to be the $\lambda$-density of $K$.

In case $\lambda_{n}=n$, the $\lambda$-density reduces to the natural density.

The number sequence $x=\left(x_{j}\right)$ is said to be $\lambda$-statistically convergent to the number $\ell$ if for each $\epsilon>0, \delta_{\lambda}\left(K_{\epsilon}\right)=0$, where $K_{\epsilon}=\left\{j \in \mathbb{N}:\left|x_{j}-\ell\right|>\epsilon\right\}$, i.e.,

$$
\lim _{n \rightarrow \infty} \frac{1}{\lambda_{n}}\left|\left\{j \in I_{n}:\left|x_{j}-\ell\right|>\epsilon\right\}\right|=0
$$

In this case, we write $s t_{\lambda}-\lim _{j} x_{j}=\ell$ and we denote the set of all $\lambda$-statistically convergent sequences by $S_{\lambda}$. This notion was extended to double sequences in [41, 42].

Remark 2.1 As in Remark 1.1, we observe that if a sequence is $(V, \lambda)$-summable to a number $\ell$, then it is also $\lambda$-statistically convergent to the same number $\ell$, but the converse need not be true. For example, let the sequence $z=\left(z_{k}\right)$ be defined by

$$
z_{k}= \begin{cases}k & \text { if } n-\left[\sqrt{\lambda_{n}}\right]+1 \leq k \leq n \\ 0 & \text { otherwise }\end{cases}
$$

where $[a]$ denotes the integer part of $a \in \mathbb{R}$. Then $x$ is $\lambda$-statistically convergent to 0 but not $(V, \lambda)$-summable.

Definition 2.1 Let $(X, \tau)$ be a locally solid Riesz space. Then a sequence $x=\left(x_{j}\right)$ in $X$ is said to be generalized statistically $\tau$-convergent (or $S_{\lambda}(\tau)$-convergent) to the number $\xi \in X$ if for every $\tau$-neighborhood $U$ of zero,

$$
\lim _{n \rightarrow \infty} \frac{1}{\lambda_{n}}\left|\left\{j \in I_{n}: x_{j}-\xi \notin U\right\}\right|=0 .
$$

In this case, we write $S_{\lambda}(\tau)-\lim x=\xi$ or $x_{j} \stackrel{S_{\lambda}(\tau)}{\longrightarrow} \xi$. 
Definition 2.2 Let $(X, \tau)$ be a locally solid Riesz space. We say that a sequence $x=\left(x_{j}\right)$ in $X$ is generalized statistically $\tau$-bounded if for every $\tau$-neighborhood $U$ of zero, there exists some $\lambda>0$ such that the set

$$
\left\{j \in \mathbb{N}: \lambda x_{j} \notin U\right\}
$$

has $\lambda$-density zero.

Theorem 2.1 Let $(X, \tau)$ be a Hausdorff locally solid Riesz space and $x=\left(x_{j}\right)$ and $y=\left(y_{k}\right)$ be two sequences in $X$. Then the following hold:

(i) If $S_{\lambda}(\tau)-\lim _{j} x_{j}=\xi_{1}$ and $S_{\lambda}(\tau)-\lim _{j} x_{j}=\xi_{2}$, then $\xi_{1}=\xi_{2}$.

(ii) If $S_{\lambda}(\tau)-\lim _{j} x_{j}=\xi$, then $S_{\lambda}(\tau)-\lim _{j} \alpha x_{j}=\alpha \xi, \alpha \in \mathbb{R}$.

(iii) If $S_{\lambda}(\tau)-\lim _{j} x_{j}=\xi$ and $S_{\lambda}(\tau)-\lim _{j} y_{j}=\eta$, then $S_{\lambda}(\tau)-\lim _{j}\left(x_{j}+y_{j}\right)=\xi+\eta$.

Proof (i) Suppose that $S_{\lambda}(\tau)-\lim _{j} x_{j}=\xi_{1}$ and $S_{\lambda}(\tau)-\lim _{j} x_{j}=\xi_{2}$. Let $U$ be any $\tau$-neighborhood of zero. Then there exists $Y \in \mathcal{N}_{\text {sol }}$ such that $Y \subseteq U$. Choose any $E \in \mathcal{N}_{\text {sol }}$ such that $E+E \subseteq Y$. We define the following sets:

$$
\begin{aligned}
& K_{1}=\left\{j \in \mathbb{N}: x_{j}-\xi_{1} \in E\right\}, \\
& K_{2}=\left\{j \in \mathbb{N}: x_{j}-\xi_{2} \in E\right\} .
\end{aligned}
$$

Since $S_{\lambda}(\tau)-\lim _{j} x_{j}=\xi_{1}$ and $S_{\lambda}(\tau)-\lim _{j} x_{j}=\xi_{2}$, we have $\delta_{\lambda}\left(K_{1}\right)=\delta_{\lambda}\left(K_{2}\right)=1$. Thus $\delta\left(K_{1} \cap K_{2}\right)=$ 1 and, in particular, $K_{1} \cap K_{2} \neq \emptyset$. Now, let $j \in K_{1} \cap K_{2}$. Then

$$
\xi_{1}-\xi_{2}=\xi_{1}-x_{j}+x_{j}-\xi_{2} \in E+E \subseteq Y \subseteq U
$$

Hence, for every $\tau$-neighborhood $U$ of zero, we have $\xi_{1}-\xi_{2} \in U$. Since $(X, \tau)$ is Hausdorff, the intersection of all $\tau$-neighborhoods $U$ of zero is the singleton set $\{\theta\}$. Thus, we get $\xi_{1}-\xi_{2}=\theta$, i.e., $\xi_{1}=\xi_{2}$.

(ii) Let $U$ be an arbitrary $\tau$-neighborhood of zero and $S_{\lambda}(\tau)-\lim _{j} x_{j}=\xi$. Then there exists $Y \in \mathcal{N}_{\text {sol }}$ such that $Y \subseteq U$ and also

$$
\lim _{n \rightarrow \infty} \frac{1}{\lambda_{n}}\left|\left\{j \in I_{n}: x_{j}-\xi \in Y\right\}\right|=1 .
$$

Since $Y$ is balanced, $x_{j}-\xi \in Y$ implies $\alpha\left(x_{j}-\xi\right) \in Y$ for every $\alpha \in \mathbb{R}$ with $|\alpha| \leq 1$. Hence, for every $n \in \mathbb{N}$, we get

$$
\begin{aligned}
\left\{j \in I_{n}: x_{j}-\xi \in Y\right\} & \subseteq\left\{j \in I_{n}: \alpha x_{j}-\alpha \xi \in Y\right\} \\
& \subseteq\left\{j \in I_{n}: \alpha x_{j}-\alpha \xi \in U\right\} .
\end{aligned}
$$

Thus, we obtain

$$
\lim _{n \rightarrow \infty} \frac{1}{\lambda_{n}}\left|\left\{j \in I_{n}: \alpha x_{j}-\alpha \xi \in U\right\}\right|=1
$$

for each $\tau$-neighborhood $U$ of zero. Now let $|\alpha|>1$ and $[|\alpha|]$ be the smallest integer greater than or equal to $|\alpha|$. There exists $E \in \mathcal{N}_{\text {sol }}$ such that $[|\alpha|] E \subseteq Y$. Since $S_{\lambda}(\tau)-\lim _{j} x_{j}=\xi$, the 
set

$$
K=\left\{j \in \mathbb{N}: x_{j}-\xi \in E\right\}
$$

has $\lambda$-density zero. Therefore, for all $n \in \mathbb{N}$ and $j \in K \cap I_{n}$, we have

$$
\left|\alpha \xi-\alpha x_{j}\right|=|\alpha|\left|\xi-x_{j}\right| \leq[|\alpha|]\left|\xi-x_{j}\right| \in[|\alpha|] E \subseteq Y \subseteq U
$$

Since the set $Y$ is solid, we have $\alpha \xi-\alpha x_{j} \in Y$. This implies that $\alpha \xi-\alpha x_{j} \in U$. Thus,

$$
\lim _{n \rightarrow \infty} \frac{1}{\lambda_{n}}\left|\left\{j \in I_{n}: \alpha x_{j}-\alpha \xi \in U\right\}\right|=1
$$

for each $\tau$-neighborhood $U$ of zero. Hence $S_{\lambda}(\tau)-\lim _{j} \alpha x_{j}=\alpha \xi$.

(iii) Let $U$ be an arbitrary $\tau$-neighborhood of zero. Then there exists $Y \in \mathcal{N}_{\text {sol }}$ such that $Y \subseteq U$. Choose $E$ in $\mathcal{N}_{\text {sol }}$ such that $E+E \subseteq Y$. Since $S_{\lambda}(\tau)-\lim _{j} x_{j}=\xi$ and $S_{\lambda}(\tau)-\lim _{j} y_{j}=\eta$, we have $\delta_{\lambda}\left(H_{1}\right)=1=\delta_{\lambda}\left(H_{2}\right)$, where

$$
\begin{aligned}
& H_{1}=\left\{j \in \mathbb{N}: x_{j}-\xi \in E\right\}, \\
& H_{2}=\left\{j \in \mathbb{N}: y_{j}-\eta \in E\right\} .
\end{aligned}
$$

Let $H=H_{1} \cap H_{2}$. Hence, we have $\delta_{\lambda}(H)=1$. For all $n \in \mathbb{N}$ and $j \in H \cap I_{n}$, we get

$$
\left(x_{j}+y_{j}\right)-(\xi+\eta)=\left(x_{j}-\xi\right)+\left(y_{j}-\eta\right) \in E+E \subseteq Y \subseteq U .
$$

Therefore,

$$
\lim _{n \rightarrow \infty} \frac{1}{\lambda_{n}}\left|\left\{j \in I_{n}:\left(x_{j}+y_{j}\right)-(\xi+\eta) \in U\right\}\right|=1 .
$$

Since $U$ is arbitrary, we have $S_{\lambda}(\tau)-\lim _{j}\left(x_{j}+y_{j}\right)=\xi+\eta$.

Theorem 2.2 Let $(X, \tau)$ be a locally solid Riesz space. If a sequence $x=\left(x_{j}\right)$ is generalized statistically $\tau$-convergent, then it is generalized statistically $\tau$-bounded.

Proof Suppose $x=\left(x_{j}\right)$ is generalized statistically $\tau$-convergent to the point $\xi \in X$ and let $U$ be an arbitrary $\tau$-neighborhood of zero. Then there exists $Y \in \mathcal{N}_{\text {sol }}$ such that $Y \subseteq U$. Let us choose $E \in \mathcal{N}_{\text {sol }}$ such that $E+E \subseteq Y$. Since $S_{\lambda}(\tau)-\lim _{j \rightarrow \infty} x_{j}=\xi$, the set

$$
K=\left\{j \in \mathbb{N}: x_{j}-\xi \notin E\right\}
$$

has $\lambda$-density zero. Since $E$ is absorbing, there exists $\lambda>0$ such that $\lambda \xi \in E$. Let $\alpha \in$ $(0, \min \{1, \lambda\})$. Since $E$ is solid and $|\alpha \xi| \leq|\lambda x|$, we have $\alpha \xi \in E$. Since $E$ is balanced, $x_{j}-\xi \in E$ implies $\alpha\left(x_{j}-\xi\right) \in E$. Then, for each $n \in \mathbb{N}$ and $j \in(\mathbb{N} \backslash K) \cap I_{n}$, we have

$$
\alpha x_{j}=\alpha\left(x_{j}-\xi\right)+\alpha \xi \in E+E \subseteq Y \subseteq U
$$


Thus

$$
\lim _{n \rightarrow \infty} \frac{1}{\lambda_{n}}\left|\left\{j \in I_{n}: \alpha x_{j} \notin U\right\}\right|=0
$$

Hence, $\left(x_{j}\right)$ is generalized statistically $\tau$-bounded.

Theorem 2.3 Let $(X, \tau)$ be a locally solid Riesz space. If $\left(x_{j}\right),\left(y_{j}\right)$ and $\left(z_{j}\right)$ are three sequences such that

(i) $x_{j} \leq y_{j} \leq z_{j}$ for all $j \in \mathbb{N}$,

(ii) $S_{\lambda}(\tau)-\lim _{j} x_{j}=\xi=S_{\lambda}(\tau)-\lim _{j} z_{j}$,

then $S_{\lambda}(\tau)-\lim _{j} y_{j}=\xi$.

Proof Let $U$ be an arbitrary $\tau$-neighborhood of zero, there exists $Y \in \mathcal{N}_{\text {sol }}$ such that $Y \subseteq U$. Choose $E \in \mathcal{N}_{\text {sol }}$ such that $E+E \subseteq Y$. From condition (ii), we have $\delta_{\lambda}(A)=1=\delta_{\lambda}(B)$, where

$$
\begin{aligned}
& A=\left\{j \in \mathbb{N}: x_{j}-\xi \in E\right\}, \\
& B=\left\{j \in \mathbb{N}: x_{j}-\xi \in E\right\} .
\end{aligned}
$$

Also, we get $\delta_{\lambda}(A \cap B)=1$, and from (i) we have

$$
x_{j}-\xi \leq y_{j}-\xi \leq z_{j}-\xi
$$

for all $j \in \mathbb{N}$. This implies that for all $n \in \mathbb{N}$ and $j \in A \cap B \cap I_{n}$, we get

$$
\left|y_{j}-\xi\right| \leq\left|x_{j}-\xi\right|+\left|z_{j}-\xi\right| \in E+E \subseteq Y \text {. }
$$

Since $Y$ is solid, we have $y_{j}-\xi \in Y \subseteq U$. Thus,

$$
\lim _{n \rightarrow \infty} \frac{1}{\lambda_{n}}\left|\left\{j \in I_{n}: y_{j}-\xi \in U\right\}\right|=1
$$

for each $\tau$-neighborhood $U$ of zero. Hence $S_{\lambda}(\tau)-\lim _{j} y_{j}=\xi$.

\section{Generalized statistically $\tau$-Cauchy and $S_{\lambda}^{*}(\tau)$-convergence}

Definition 3.1 Let $(X, \tau)$ be a locally solid Riesz space. A sequence $x=\left(x_{j}\right)$ in $X$ is generalized statistically $\tau$-Cauchy if for every $\tau$-neighborhood $U$ of zero there exists $p \in \mathbb{N}$ such that the set

$$
\left\{j \in \mathbb{N}: x_{j}-x_{p} \notin U\right\}
$$

has $\lambda$-density zero.

Theorem 3.1 Let $(X, \tau)$ be a locally solid Riesz space. If a sequence $x=\left(x_{j}\right)$ is generalized statistically $\tau$-convergent, then it is generalized statistically $\tau$-Cauchy. 
Proof Suppose that $S_{\lambda}(\tau)-\lim _{j} x_{j}=\xi$. Let $U$ be an arbitrary $\tau$-neighborhood of zero, there exists $Y \in \mathcal{N}_{\text {sol }}$ such that $Y \subseteq U$. Choose $E \in \mathcal{N}_{\text {sol }}$ such that $E+E \subseteq Y$. By generalized statistical $\tau$-convergence to $\xi$, there is $p \in \mathbb{N}$ with $\xi-x_{p} \in E$ and

$$
\lim _{n \rightarrow \infty} \frac{1}{\lambda_{n}}\left|\left\{j \in I_{n}: x_{j}-\xi \notin E\right\}\right|=0 .
$$

Also, for all $n \in \mathbb{N}$ and $j \in(\mathbb{N} \backslash K) \cap I_{n}$, where

$$
K=\left\{j \in \mathbb{N}: x_{j}-\xi \notin E\right\},
$$

we have

$$
x_{j}-x_{p}=x_{j}-\xi+\xi-x_{p} \in E+E \subseteq Y \subseteq U
$$

and $\delta_{\lambda}(K)=0$. Therefore the set

$$
\left\{j \in \mathbb{N}: x_{j}-x_{p} \notin U\right\} \subseteq K \cap I_{n}
$$

for all $n \in \mathbb{N}$. For every $\tau$-neighborhood $U$ of zero there exists $p \in \mathbb{N}$ such that the set $\left\{j \in \mathbb{N}: x_{j}-x_{p} \notin U\right\}$ has $\lambda$-density zero. Hence $\left(x_{j}\right)$ is generalized statistically $\tau$-Cauchy.

Now we define another type of convergence in locally solid Riesz spaces.

Definition 3.2 A sequence $\left(x_{j}\right)$ in a locally solid Riesz space $(X, \tau)$ is said to be $S_{\lambda}^{*}(\tau)$ convergent to $\xi \in X$ if there exists an index set $K=\left\{j_{n}\right\} \subseteq \mathbb{N}, n=1,2, \ldots$, with $\delta_{\lambda}(K)=1$ such that $\lim _{n \rightarrow \infty} x_{j_{n}}=\xi$. In this case, we write $\xi=S_{\lambda}^{*}(\tau)-\lim x$.

Theorem 3.2 A sequence $x=\left(x_{j}\right)$ in a locally solid Riesz space $(X, \tau)$ is generalized statistically $\tau$-convergent to a number $\xi$ if it is $S_{\lambda}^{*}(\tau)$-convergent to $\xi$.

Proof Let $U$ be an arbitrary $\tau$-neighborhood of $\xi$. Since $x=\left(x_{j}\right)$ is $S_{\lambda}^{*}(\tau)$-convergent to $\xi$, there is an index set $K=\left\{j_{n}\right\} \subseteq \mathbb{N}, n=1,2, \ldots$, with $\delta_{\lambda}(K)=1$ and $j_{0}=j_{0}(U)$, such that $j \geq j_{0}$ and $j \in K$ imply $x_{j}-\xi \in U$. Then

$$
K_{U}=\left\{j \in \mathbb{N}: x_{j}-\xi \notin U\right\} \subseteq \mathbb{N}-\left\{j_{N+1}, j_{N+2}, \ldots\right\} .
$$

Therefore $\delta_{\lambda}\left(K_{U}\right)=0$. Hence $x$ is generalized statistically $\tau$-convergent to $\xi$.

Note that the converse holds for a first countable space.

Recall that a topological space is first countable if each point has a countable (decreasing) local base.

Theorem 3.3 Let $(X, \tau)$ be a first countable locally solid Riesz space. If a sequence $x=\left(x_{j}\right)$ is generalized statistically $\tau$-convergent to a number $\xi$, then it is $S_{\lambda}^{*}(\tau)$-convergent to $\xi$. 
Proof Let $x$ be generalized statistically $\tau$-convergent to a number $\xi$. Fix a countable local base $U_{1} \supset U_{2} \supset U_{3} \supset \cdots$ at $\xi$. For each $i \in \mathbb{N}$, put

$$
K_{i}=\left\{j \in \mathbb{N}: x_{j}-\xi \notin U_{i}\right\}
$$

By hypothesis, $\delta_{\lambda}\left(K_{i}\right)=0$ for each $i$. Since the ideal $\mathcal{I}$ of all subsets of $\mathbb{N}$ having $\lambda$-density zero is a $P$-ideal (see, for instance, [43]), then there exists a sequence of sets $\left(J_{i}\right)_{i}$ such that the symmetric difference $K_{i} \Delta J_{i}$ is a finite set for any $i \in \mathbb{N}$ and $J:=\bigcup_{i=1}^{\infty} J_{i} \in \mathcal{I}$.

Let $K=\mathbb{N} \backslash J$, then $\delta_{\lambda}(K)=1$. In order to prove the theorem, it is enough to check that $\lim _{j \in K} x_{j}=\xi$.

Let $i \in \mathbb{N}$. Since $K_{i} \Delta J_{i}$ is finite, there is $j_{i} \in \mathbb{N}$, without loss of generality, with $j_{i} \in K, j_{i}>i$, such that

$$
\left(\mathbb{N} \backslash J_{i}\right) \cap\left\{j \in \mathbb{N}: j \geq j_{i}\right\}=\left(\mathbb{N} \backslash K_{i}\right) \cap\left\{j \in \mathbb{N}: j \geq j_{i}\right\} .
$$

If $j \in K$ and $j \geq j_{i}$, then $j \notin J_{i}$, and by (1), $j \notin K_{i}$. Thus $x_{j}-\xi \in U_{i}$. So, we have proved that for all $i \in \mathbb{N}$, there is $j_{i} \in K, j_{i}>i$, with $x_{j}-\xi \in U_{i}$ for every $j \geq j_{i}$ : without loss of generality, we can suppose $j_{i+1}>j_{i}$ for every $i \in \mathbb{N}$. The assertion follows taking into account that the $U_{i}$ 's form a countable local base at $\xi$.

\section{Conclusion}

Recently, statistical convergence has been established as a better option than ordinary convergence. It is found very interesting that some results on sequences, series and summability can be proved by replacing the ordinary convergence by statistical convergence; and further, through some examples, where some efforts are required, we can show that the results for statistical convergence happen to be stronger than those proved for ordinary convergence (e.g., [44-49]). This notion has also been defined and studied in different setups. In this paper, we have studied this notion through de la Vallée-Poussin mean in a locally solid Riesz space to deal with the convergence problems in a broader sense.

Competing interests

The authors declare that they have no competing interests.

Authors' contributions

The authors contributed equally and significantly in writing this paper. All authors read and approved the final manuscript.

\section{Author details}

'Department of Mathematics, Faculty of Science, King Abdulaziz University, P.O. Box 80203, Jeddah, 21589, Saudi Arabia.

${ }^{2}$ Department of Mathematics, Aligarh Muslim University, Aligarh, 202002, India.

\section{Acknowledgements}

The authors gratefully acknowledge the financial support from King Abdulaziz University, Jeddah, Saudi Arabia.

Received: 16 November 2012 Accepted: 1 March 2013 Published: 21 March 2013

References

1. Steinhaus, H: Sur la convergence ordinaire et la convergence asymptotique. Colloq. Math. 2, $73-74$ (1951)

2. Fast, H: Sur la convergence statistique. Colloq. Math. 2, 241-244 (1951)

3. Çakalli, H: Lacunary statistical convergence in topological groups. Indian J. Pure Appl. Math. 26(2), $113-119$ (1995)

4. Cakalli, H: On statistical convergence in topological groups. Pure Appl. Math. Sci. 43, 27-31 (1996)

5. Çakalli, H, Khan, MK: Summability in topological spaces. Appl. Math. Lett. 24, 348-352 (2011)

6. Çakalli, H, Savaş, E: Statistical convergence of double sequence in topological groups. J. Comput. Anal. Appl. 12(2), 421-426 (2010) 
7. Edely, OHH, Mursaleen, M: On statistical A-summability. Math. Comput. Model. 49, 672-680 (2009)

8. Fridy, JA: On statistical convergence. Analysis 5, 301-313 (1985)

9. Karakuş, S, Demirci, K: Statistical convergence of double sequences on probabilistic normed spaces. Int. J. Math. Math. Sci. 2007, Article ID 14737 (2007)

10. Karakuş, S, Demirci, K, Duman, O: Statistical convergence on intuitionistic fuzzy normed spaces. Chaos Solitons Fractals 35, 763-769 (2008)

11. Maddox, IJ: Statistical convergence in a locally convex space. Math. Proc. Camb. Philos. Soc. 104, 141-145 (1988)

12. Mohiuddine, SA, Aiyub, M: Lacunary statistical convergence in random 2-normed spaces. Appl. Math. Inf. Sci. 6(3), 581-585 (2012)

13. Mohuiddine, SA, Alotaibi, A, Alsulami, SM: Ideal convergence of double sequences in random 2-normed spaces. Adv. Differ. Equ. 2012, 149 (2012)

14. Mohiuddine, SA, Danish Lohani, QM: On generalized statistical convergence in intuitionistic fuzzy normed space. Chaos Solitons Fractals 42, 1731-1737 (2009)

15. Mohiuddine, SA, Savaş, E: Lacunary statistically convergent double sequences in probabilistic normed spaces. Ann Univ. Ferrara 58, 331-339 (2012)

16. Mohiuddine, SA, Sevli, H, Cancan, M: Statistical convergence in fuzzy 2-normed space. J. Comput. Anal. Appl. 12(4), 787-798 (2010)

17. Mohiuddine, SA, Sevli, H, Cancan, M: Statistical convergence of double sequences in fuzzy normed spaces. Filomat 26(4), 673-681 (2012)

18. Mursaleen, M: On statistical convergence in random 2-normed spaces. Acta Sci. Math. 76, 101-109 (2010)

19. Mursaleen, M, Alotaibi, A: On /-convergence in random 2-normed spaces. Math. Slovaca 61(6), 933-940 (2011)

20. Mursaleen, M, Edely, OHH: Generalized statistical convergence. Inf. Sci. 162, 287-294 (2004)

21. Mursaleen, M, Edely, OHH: Statistical convergence of double sequences. J. Math. Anal. Appl. 288, 223-231 (2003)

22. Mursaleen, $\mathrm{M}$, Edely, OHH: On the invariant mean and statistical convergence. Appl. Math. Lett. 22, 1700-1704 (2009)

23. Mursaleen, M, Mohiuddine, SA: Statistical convergence of double sequences in intuitionistic fuzzy normed spaces. Chaos Solitons Fractals 41, 2414-2421 (2009)

24. Mursaleen, M, Mohiuddine, SA: On lacunary statistical convergence with respect to the intuitionistic fuzzy normed space. J. Comput. Appl. Math. 233, 142-149 (2009)

25. Mursaleen, $\mathrm{M}$, Mohiuddine, SA: On ideal convergence of double sequences in probabilistic normed spaces. Math. Rep. 12(64)(4), 359-371 (2010)

26. Mursaleen, M, Mohiuddine, SA: On ideal convergence in probabilistic normed spaces. Math. Slovaca 62, 49-62 (2012)

27. Mursaleen, $\mathrm{M}$, Mohiuddine, SA, Edely, OHH: On the ideal convergence of double sequences in intuitionistic fuzzy normed spaces. Comput. Math. Appl. 59, 603-611 (2010)

28. Savaş, E, Mohiuddine, SA: $\bar{\lambda}$-statistically convergent double sequences in probabilistic normed spaces. Math. Slovaca 62(1), 99-108 (2012)

29. Savaş, E, Mursaleen, M: On statistically convergent double sequences of fuzzy numbers. Inf. Sci. 162, 183-192 (2004)

30. Di Maio, G, Kočinac, LDR: Statistical convergence in topology. Topol. Appl. 156, $28-45$ (2008)

31. Albayrak, H, Pehlivan, S: Statistical convergence and statistical continuity on locally solid Riesz spaces. Topol. Appl. 159, 1887-1893 (2012)

32. Mohiuddine, SA, Alghamdi, MA: Statistical summability through a lacunary sequence in locally solid Riesz spaces J. Inequal. Appl. 2012, 225 (2012)

33. Mohiuddine, SA, Alotaibi, A, Mursaleen, M: Statistical convergence of double sequences in locally solid Riesz spaces. Abstr. Appl. Anal. 2012, Article ID 719729 (2012)

34. Zaanen, AC: Introduction to Operator Theory in Riesz Spaces. Springer, Berlin (1997)

35. Aliprantis, CD, Burkinshaw, O: Locally Solid Riesz Spaces with Applications to Economics, 2nd edn. Am. Math. Soc., Providence (2003)

36. Roberts, GT: Topologies in vector lattices. Math. Proc. Camb. Philos. Soc. 48, 533-546 (1952)

37. Mursaleen, M: $\lambda$-statistical convergence. Math. Slovaca 50, 111-115 (2000)

38. Çolak, R, Bektaș, CA: $\lambda$-statistical convergence of order $\alpha$. Acta Math. Sci., Ser. B 31(3), $953-959$ (2011)

39. Edely, $\mathrm{OHH}$, Mohiuddine, SA, Noman, AK: Korovkin type approximation theorems obtained through generalized statistical convergence. Appl. Math. Lett. 23, 1382-1387 (2010)

40. de Malafosse, B, Rakočević, V: Matrix transformation and statistical convergence. Linear Algebra Appl. 420, 377-387 (2007)

41. Mursaleen, M, Çakan, C, Mohiuddine, SA, Savaş, E: Generalized statistical convergence and statistical core of double sequences. Acta Math. Sin. Engl. Ser. 26, 2131-2144 (2010)

42. Kumar, V, Mursaleen, $\mathrm{M}$ : On $(\lambda, \mu)$-statistical convergence of double sequences on intuitionistic fuzzy normed spaces. Filomat 25(2), 109-120 (2011)

43. Farah, I: Analytic Quotients: Theory of Liftings for Quotients over Analytic Ideals on the Integers. Mem. Amer. Math. Soc., vol. 148 (2000)

44. Caserta, A, Kočinac, LDR: On statistical exhaustiveness. Appl. Math. Lett. 25, 1447-1451 (2012)

45. Caserta, A, Di Maio, G, Kočinac, LDR: Statistical convergence in function spaces. Abstr. Appl. Anal. 2011, Article ID 420419 (2011)

46. Mohiuddine, SA: An application of almost convergence in approximation theorems. Appl. Math. Lett. 24, 1856-1860 (2011)

47. Mohiuddine, SA, Alotaibi, A: Statistical convergence and approximation theorems for functions of two variables. J. Comput. Anal. Appl. 15(2), 218-223 (2013)

48. Mohiuddine, SA, Alotaibi, A, Mursaleen, M: Statistical summability $(C, 1)$ and a Korovkin type approximation theorem. J. Inequal. Appl. 2012, 172 (2012)

49. Srivastava, HM, Mursaleen, M, Khan, A: Generalized equi-statistical convergence of positive linear operators and associated approximation theorems. Math. Comput. Model. 55, 2040-2051 (2012) 
doi:10.1186/1687-1847-2013-66

Cite this article as: Mohiuddine et al.: Statistical convergence through de la Vallée-Poussin mean in locally solid Riesz spaces. Advances in Difference Equations 2013 2013:66.

Submit your manuscript to a SpringerOpen ${ }^{\circ}$ journal and benefit from:

- Convenient online submission

- Rigorous peer review

- Immediate publication on acceptance

Open access: articles freely available online

- High visibility within the field

- Retaining the copyright to your article

Submit your next manuscript at $\boldsymbol{s p r i n g e r o p e n . c o m ~}$ 